\title{
Paneth cells and inflammation dance together in Crohn's disease
}

\author{
Arthur Kaser ${ }^{1}$, Richard S Blumberg ${ }^{2}$ \\ ${ }^{1}$ Division of Gastroenterology, Department of Medicine, Innsbruck Medical University, Anichstrasse 35, 6020 Innsbruck, Austria; \\ ${ }^{2}$ Division of Gastroenterology, Department of Medicine, Brigham and Women's Hospital, Harvard Medical School, 75 Francis Street, \\ Boston, MA 02115 USA. Correspondence: rblumberg@partners.org \\ Cell Research (2008) 18:1160-1162. doi: 10.1038/cr.2008.312; published online 1 December 2008
}

Inflammatory Bowel Disease (IBD) is a dysregulated response of the immune system associated with intestinal tissues to the commensal microbiota in a genetically susceptible host. The understanding of the genetic basis of IBD has been significantly expanded with the advent of genome-wide association studies (GWAS) which has resulted in the identification of more than 30 loci that are associated with Crohn's disease (CD) and ulcerative colitis (UC). As anticipated, this unbiased approach of gene discovery has both confirmed previously established disease related pathways and led to new hypotheses of IBD pathogenesis that are currently being tested in biologic model systems to establish the precise mechanisms of action involved. One such potential pathway that was unexpectedly identified is that associated with autophagy; a cell biologic pathway important in the clearance of microbes and cellular proteins and organelles.

Employing a GWAS of non-synonymous single nucleotide polymorphisms (nsSNPs) Schreiber and colleagues first identified and confirmed ATG16L1, a putative human orthologue of a yeast autophagy gene, as a susceptibility factor specifically for CD [1]. Haplotype and regression analysis further identified a single coding SNP ( $r s 2241880$, T300A) of unknown function that conferred virtually all the disease risk exerted by the ATG16L1 locus [1]. Reproduction by other groups of the association of ATG16L1 with CD $[2,3]$ together with the identification of $I R G M$ and possibly $L R K K 2$, additional autophagy genes, as risk factors for $\mathrm{CD}[3,4]$, has strongly implicated autophagy as a pathway unique to CD but not UC. However, it has remained to be defined how these ATG16L1 genotypes confer the phenotypic development of CD.

Two papers published in Nature online on October 5, 2008, shed some light on how Atg1611 and autophagy may contribute to the development of CD $[5,6]$. Using two different genetrap-mediated hypomorphic disruptions of Atg16l1 as well as mice with $\operatorname{Atg} 5$ conditionally deleted in the intestinal epithelium, Cadwell et al. report that Atg1611 is indeed a bona fide autophagy protein as predicted from its homology to the yeast autophagy protein ATG16. By using a variety of morphological measures, they report that Atg1611 and Atg5 play a major role in the formation of Paneth cell $(\mathrm{Pc})$ granules that are rich in antimicrobial peptides and involved in host defense against infection and presumably the management of commensalism. Moreover, transcriptional profiling of Pc in hypomorphic Atg16l1 mice showed increased expression of genes involved in peroxisome proliferator-activated receptor-signaling as well as leptin and adiponectin, adipocy- tokines that have been linked to intestinal inflammation and are increased in CD [5]. Most interestingly, in patients homozygous for the disease-associated ATG16L1 variant (T300A), the authors report morphologic Pc abnormalities and increased leptin expression reminiscent of those observed in their hypomorphic Atg16l1 mouse models [5]. In the second paper, Saitoh et al. report that Atg1611 regulates endotoxin-induced inflammosome activation [6]. Atg1611 deficiency was shown to disrupt the recruitment of the Atg12-Atg5 conjugate to the isolation membrane resulting in a loss of microtubule-associated protein 1 light chain 3 (LC3; homologue of yeast Atg8) conjugation to phosphatidylethanolamine [6]; an essential step in autophagosome formation. As a consequence, both autophagosome formation and degradation of long-lived proteins were severely impaired as shown by accumulation of the adaptor protein sequestosome (SQSTM1/P62) [6]. Stimulation of Atg1611-deficient macrophages derived from fetal liver with the toll-like receptor 4 (TLR4) ligand lipopolysaccharide (LPS) resulted in increased secretion of IL- $1 \beta$ and IL-18 via TRIF (Toll/IL-1 receptor domain-containing adaptor inducing IFN $\beta$ )-dependent activation of caspase-1 [6]. Furthermore, simple chemical disruption of autophagy enhanced IL- $1 \beta$ production by primary wild-type macrophages 
induced by LPS demonstrating the fundamental association between these two biologic pathways. Chimeric mice that lacked Atg1611 in hematopoietic cells were highly susceptible to dextran sodium sulphate (DSS) colitis, a colitis known to be initiated by the dropout of intestinal epithelial cells and macrophage activation. Finally, anti-IL-1 $\beta$ and anti-IL-18 antibodies ameliorated DSS colitis in chimeric Atg16l1 mice suggesting that inappropriate secretion of these cytokines by dysfunctional hematopoietic cells accounts for the phenotype in this model [6].

These first mechanistic data on Atg1611 and autophagy and how it might function in the development of CD highlight a remarkable convergence of several IBD-associated genes on two common themes, Paneth cells and the IL-1 pathway [7-9]. Nod2 ${ }^{--}$mice, for example, have been previously shown to exhibit decreased expression of specific cryptdins ( $\alpha$-defensins) associated with Pc and a severe defect in handling an orally, but not parenterally, administered pathogen, Listeria monocytogenes [7]. Given the important antimicrobial function of $\mathrm{Pc}$, it is reasonable to conclude that this defect in Pc expression of cryptdins due to NOD2-deficiency may predispose to an inability to manage luminal bacteria as modelled through responses to L. monocytogenes. However, macrophages from $\mathrm{Nod}^{-/-}$mice also exhibit decreased NFKB and MAP kinase activation which is accompanied by decreased IL- 6 and TNF $\alpha$ secretion upon stimulation with muramyl dipeptide (MDP), a ligand of NOD2 [7], suggesting that NOD2 may also regulate the ability of cell types in addition to Pc to oversee the management of luminal microbiota.

It is notable that, in contrast to Nod2 ${ }^{-/}$mice, Atg16l1-hypomorphic mice did not exhibit significant deficiencies in handling L. monocytogenes despite remarkable alterations in Pc granule structure and composition [5]. One interpretation of these results is that alterations in autophagy due to defective ATG16L1 function do not affect the antimicrobial activities of $\mathrm{Pc}$ through their ability to secrete cryptdins. This may not be the case for other cell types affected by abnormal ATG16L1 function. For example, ATG16L1 T300A varianttransfected absorptive epithelial cells exhibit impaired capture of internalized Salmonella $s p$. within autophagosomes, while not showing any differences in basal autophagy [10]. Thus, ATG16L1 may affect the antimicrobial activity of epithelial cell types active in bacterial clearance through autophagosome formation but not necessarily through antimicrobial peptide secretion.

It is interesting in this regard that, in contrast to normal handling of L. monocytogenes, the Pc of Atg16l1-hypomorphic mice exhibit an inflammatory repertoire [5]. Transcriptional profiling of Atg16l1-hypomorphic Pc showed upregulation of inflammatory mediators including leptin and adiponectin, known to be increased in human $\mathrm{CD}$ and capable of driving inflammatory adaptive immune responses. In a similar manner, as summarized above, Atg1611deficient macrophages are also hyperinflammatory in a pathway that involves hypersecretion of IL-1 $\beta$ and IL-18 [6]. Hypomorphic NOD2 variants have also been reported to be hyper-inflammatory in a pathway that involves IL- $1 \beta$. Maeda et al. reported a knock-in mouse model [8] which expressed one of the CDassociated NOD2 variants, 3020insC (2939ins C in mouse). Nod2 $2^{2939 i n s C}$ mice exhibit increased $\mathrm{NF \kappa B}$ activation in response to MDP and more efficient processing and secretion of IL-1 $1 \beta$ [8]. This was accompanied by increased susceptibility to DSS colitis in Nod2 $2^{2939 \text { ins }}$ mice compared to wild-type controls, a phenotype that was abrogated by administration of IL-1 receptor antagonist as well as depletion of the commensal microbial flora through administration of antibiotics [8]. While the cell type responsible for this hyperinflammatory activity is unknown and may be an epithelial cell, including potentially the $\mathrm{Pc}$, it is interesting that two groups [11, 12] have reported a hyper-inflammatory phenotype of myeloid cells in response to Toll like receptor signalling in the absence of normal NOD2 function.

Interestingly, neither Atg16l1-hypomorphic mice [5], Atg16l1-deficient fetal liver chimeric mice [6], Nod2 $2^{-1}$ mice [7] nor Nod2 ${ }^{2939 \text { insC }^{2}}$ mice [8] develop spontaneous intestinal inflammation. Moreover, Nod2 $2^{-/}$mice do not exhibit an increased susceptibility to DSS colitis [7]. These observations are consistent with an inability of Pc abnormalities alone to induce intestinal inflammation. Specifically, Pc depletion [13] or an inability to activate $\alpha$-defensins [14] are not associated with spontaneous intestinal inflammation. This strongly argues against a dominant inflammation-inducing effect due to alterations in the microflora alone. Rather, other recent data support a "two-hit hypothesis" wherein host (or potentially environmentally)-mediated alterations in the intestinal microbiota may only induce dysregulated intestinal inflammation characteristic of CD (and IBD) when present together with a tendency to hyperrespond to microbial stimuli. This notion is revealed by an examination of X-box binding protein 1 (XBP1) which has recently been identified as a genetic risk factor for the development of both CD and UC [9]. XBP1 is an important regulator of the unfolded protein response in the setting of endoplasmic reticulum (ER) stress [15].

Mice with a specific deletion of Xbp1 in the intestinal epithelium uniquely develop spontaneous small bowel enteritis, with hallmarks of human IBD such as leukocyte infiltration and crypt abscesses, and increased susceptibility to DSS colitis [9]. The predominant cell type affected in these conditionally deleted $\mathrm{Xbp1}^{-/-}$mice is the Pc which exhibit a severe structural defect that is characterized by a condensed ER and only a few small granules, diminished secretion of antimicrobial peptides and 
antimicrobial activity of the crypts together with an inability to manage mucosal infection with L. monocytogenes [9]. Among the four intestinal epithelial cell lineages, Pc are unique in their high secretory activity making them especially vulnerable to abnormalities in ER stress mechanisms $[9,15]$. In addition to this profound defect in the antimicrobial activity of the epithelium through Pc dysfunction, IECs (intestinal epithelial cells) that are deficient in Xbp1 function exhibit heightened Jun kinase activation in response to inflammatory cytokines (e.g. TNF $\alpha$ ) and microbial products known to bind Toll like receptors (e.g. flagellin) [9]. These studies with XBP1, NOD2 and ATG16L1 reveal that the intestinal inflammation associated with IBD may arise primarily from defects within the epithelium and specifically the Pc but only when both of the two major drivers of IBD are affected: the ability to regulate the composition of the intestinal microbiota and the responsiveness of the epithelium (and/or the hematopoietic cells) to inflammatory microbial signals.

\section{Aknowledgment}

This article is supported by the Medizinische Forschungsförderung Innsbruck (A.K.), National Institutes of Health DK44319, DK51362 and DK53056 (R.S.B.), and the Crohn's and Colitis Foundation of America (R.S.B.).

\section{References}

1 Hampe J, Franke A, Rosenstiel P, et al. A genome-wide association scan of nonsynonymous SNPs identifies a susceptibility variant for Crohn disease in ATG16L1. Nat Genet 2007; 39:207211.

2 Rioux JD, Xavier RJ, Taylor KD, et al. Genome-wide association study identifies new susceptibility loci for Crohn disease and implicates autophagy in disease pathogenesis. Nat Genet 2007; 39:596-604.

3 The Wellcome Trust Case Control Consortium. Genome-wide association study of 14,000 cases of seven common diseases and 3,000 shared controls. Nature 2007; 447:661-678.

4 Barrett JC, Hansoul S, Nicolae DL, et al. Genome-wide association defines more than 30 distinct susceptibility loci for Crohn's disease. Nat Genet 2008; 40:955-962.

5 Cadwell K, Liu JY, Brown SL, et al. A key role for autophagy and the autophagy gene Atg1611 in mouse and human intestinal Paneth cells. Nature 2008; doi:10.1038/nature07416.

6 Saitoh T, Fujita N, Jang MH. et al. Loss of the autophagy protein Atg16L1 enhances endotoxin-induced IL-1beta production. Nature 2008; doi:10.1038/ nature 07383 .

7 Kobayashi KS, Chamaillard M, Ogura Y, et al. Nod2-dependent regulation of innate and adaptive immunity in the intestinal tract. Science 2005; 307:731734.
8 Maeda S, Hsu LC, Liu H, et al. Nod2 mutation in Crohn's disease potentiates NF-kappaB activity and IL-1beta processing. Science 2005; 307:734738.

9 Kaser A, Lee AH, Franke A, et al. XBP1 links ER stress to intestinal inflammation and confers genetic risk for human inflammatory bowel disease. Cell 2008; 134:743-756.

10 Kuballa P, Huett A, Rioux JD, Daly MJ, Xavier RJ. Impaired autophagy of an intracellular pathogen induced by a Crohn's disease associated ATG16L1 variant. PLoS ONE 2008; 3:e3391.

11 Watanabe T, Kitani A, Murray PJ, Strober W. NOD2 is a negative regulator of Toll-like receptor 2-mediated Thelper type 1 responses. Nat Immunol 2004; 5:800-808.

12 Hedl M, Li J, Cho JH, Abraham C. Chronic stimulation of Nod2 mediates tolerance to bacterial products. Proc Natl Acad Sci USA 2007; 104:1944019445.

13 Garabedian EM, Roberts LJ, McNevin MS, Gordon JI. Examining the role of Paneth cells in the small intestine by lineage ablation in transgenic mice. $J$ Biol Chem 1997; 272: 23729-23740.

14 Wilson CL, Ouellette AJ, Satchell DP, et al. Regulation of intestinal alphadefensin activation by the metalloproteinase matrilysin in innate host defense. Science 1999; 286:113-7.

15 Todd DJ, Lee AH, Glimcher LH. The endoplasmic reticulum stress response in immunity and autoimmunity. Nat Rev Immunol 2008; 8:663-674. 